\title{
Interval Oscillation Criteria for Second-Order Forced Functional Dynamic Equations on Time Scales
}

\author{
Shao-Yan Zhang ${ }^{1}$ and Qi-Ru Wang ${ }^{2}$ \\ ${ }^{1}$ Department of Mathematics, Guangdong University of Finance, Guangzhou 510520, China \\ ${ }^{2}$ School of Mathematics \& Computational Science, Sun Yat-Sen University, Guangzhou 510275, China \\ Correspondence should be addressed to Qi-Ru Wang; mcswqr@mail.sysu.edu.cn
}

Received 26 November 2013; Revised 15 January 2014; Accepted 18 January 2014; Published 18 March 2014

Academic Editor: Delfim F. M. Torres

Copyright (c) 2014 S.-Y. Zhang and Q.-R. Wang. This is an open access article distributed under the Creative Commons Attribution License, which permits unrestricted use, distribution, and reproduction in any medium, provided the original work is properly cited.

\begin{abstract}
This paper is concerned with oscillation of second-order forced functional dynamic equations of the form $\left(r(t)\left(x^{\Delta}(t)\right)^{\gamma}\right)^{\Delta}+$ $\sum_{i=0}^{n} q_{i}(t)\left|x\left(\delta_{i}(t)\right)\right|^{\alpha_{i}}$ sgn $x\left(\delta_{i}(t)\right)=e(t)$ on time scales. By using a generalized Riccati technique and integral averaging techniques, we establish new oscillation criteria which handle some cases not covered by known criteria.
\end{abstract}

\section{Introduction}

The theory of time scales was introduced by Stefan Hilger in his Ph.D. thesis in 1988 in order to unify the continuous and discrete analysis. Not only can this theory of the so-called "dynamic equations" unify theories of differential equations and difference equations but also it can extend these classical cases to cases "in between," for example, to the so-called $q$ difference equations. A time scale $\mathbb{T}$ is an arbitrary nonempty closed subset of the real numbers $\mathbb{R}$ with the topology and ordering inherited form $\mathbb{R}$, and the cases when this time scale is equal to $\mathbb{R}$ or to the integers $\mathbb{Z}$ represent the classical theories of differential and difference equations. Of course many other interesting time scales exist, and they give rise to plenty of applications. In recent years, there has been much research activity concerning the oscillation and nonoscillation of solutions of various dynamic equations on time scales, and we refer the readers to [1-20].

In 2006, Saker [1] provided sufficient conditions for the boundedness of the solutions of forced dynamic equations of the form:

$$
x^{\Delta \Delta}(t)+q^{\sigma}(t) f(x(t))=e(t) .
$$

In 2007, Sun and Wong [2] considered interval oscillation of second-order forced ordinary differential equations with mixed nonlinearities:

$$
\begin{aligned}
& \left(p(t) x^{\prime}(t)\right)^{\prime}+q(t) x(t) \\
& \quad+\sum_{i=1}^{n} q_{i}(t)|x(t)|^{\alpha_{i}} \operatorname{sgn} x(t)=e(t),
\end{aligned}
$$

where $\alpha_{1}>\cdots>\alpha_{m}>1>\alpha_{m+1}>\cdots>\alpha_{n}$.

In 2008, Erbe et al. [3] established oscillation criteria for the forced second-order nonlinear dynamic equation:

$$
\left(p(t) x^{\Delta}(t)\right)^{\Delta}+q(t)\left|x^{\sigma}(t)\right|^{\gamma} \operatorname{sgn} x^{\sigma}(t)=e(t), \quad \gamma \geq 1 .
$$

In 2009, Li and Chen [4] considered oscillation of second-order functional differential equations with mixed nonlinearities:

$$
\begin{aligned}
& \left(p(t) x^{\prime}(t)\right)^{\prime}+q(t) x(t-\tau) \\
& \quad+\sum_{i=1}^{n} q_{i}(t)|x(t-\tau)|^{\alpha_{i}} \operatorname{sgn} x(t-\tau)=e(t), \quad \tau \geq 0 .
\end{aligned}
$$


In 2010, Lin et al. [5] considered forced oscillation of second-order half-linear dynamic equations on time scales:

$$
\left(r(t)\left[x^{\Delta}(t)\right]^{\alpha}\right)^{\Delta}+p(t) x^{\alpha}(\sigma(t))=f(t),
$$

where $\alpha$ is a quotient of odd positive integers. Also, Erbe et al. [6] obtained some interval oscillation criteria for forced second order nonlinear delay dynamic equations with oscillatory potential of the form:

$$
\left(r(t) x^{\Delta}(t)\right)^{\Delta}+q(t) f(x(\tau(t)))=e(t) .
$$

In 2011, Hassan et al. [7] discussed oscillation of the following forced second-order differential equations with mixed nonlinearities:

$$
\begin{aligned}
& \left(a(t)\left(x^{\prime}(t)\right)^{\gamma}\right)^{\prime}+p_{0}(t) x^{\gamma}(t) \\
& +\sum_{i=1}^{n} p_{i}(t)|x(t)|^{\alpha_{i}} \operatorname{sgn} x(t)=e(t), \\
& \left(a(t)\left(x^{\prime}(t)\right)^{\gamma}\right)^{\prime}+p_{0}(t) x^{\gamma}\left(g_{0}(t)\right) \\
& \quad+\sum_{i=1}^{n} p_{i}(t)\left|x\left(g_{i}(t)\right)\right|^{\alpha_{i}} \operatorname{sgn} x\left(g_{i}(t)\right)=e(t),
\end{aligned}
$$

where $\gamma$ is a quotient of odd positive integers, $\alpha_{i}>0, i=$ $1,2, \ldots, n$ and $\alpha_{1}>\cdots>\alpha_{m}>\gamma>\alpha_{m+1}>\cdots>\alpha_{n}$ with $a(t), e(t), p_{i}(t) \in C\left(\left[t_{0}, \infty\right), \mathbb{R}\right), a(t)>0, g_{i}(t): \mathbb{R} \rightarrow \mathbb{R}$ is positive and nondecreasing continuous and $\lim _{t \rightarrow \infty} g_{i}(t)=$ $\infty$ for $i=1,2, \ldots, n$. The authors established some sufficient conditions for the oscillation of (7), which did not assume that $e(t)$ and $p_{i}(t)(i=1,2, \ldots, n)$ are of definite sign.

In 2013, Anderson and Saker [8] establish some oscillation criteria for the second-order nonlinear Emden-Fowler functional dynamic equation with oscillatory potential and forcing term on time scales of the form:

$$
\left(r x^{\Delta}\right)^{\Delta}(t)+q(t)|x(\tau(t))|^{\gamma} x(\tau(t))=e(t), \quad t \in \mathbb{T},
$$

where $\mathbb{T}$ is a time scale unbounded above, $\gamma>1$, the potentials $r$ and $q$ and the forcing function $e$ are right dense continuous with $r>0$, and $\tau: \mathbb{T} \rightarrow \mathbb{T}$ satisfies $\lim _{t \rightarrow \infty} \tau(t)=$ $\infty$. They also did not assume that $e$ and $q$ are of definite sign.

In this paper, motivated by [1-8] and others, we study the second-order nonlinear dynamic equation:

$$
\left(r(t)\left(x^{\Delta}(t)\right)^{\gamma}\right)^{\Delta}+\sum_{i=0}^{n} q_{i}(t)\left|x\left(\delta_{i}(t)\right)\right|^{\alpha_{i}} \operatorname{sgn} x\left(\delta_{i}(t)\right)=e(t)
$$

on a time scale $\mathbb{T}$, where $e(t), q_{i}(t) \in C_{\text {rd }}(\mathbb{T}, \mathbb{R}), \gamma>0$ is a quotient of odd positive integers, $\alpha_{0}=\gamma$, and $\left\{\alpha_{1}, \alpha_{2}, \ldots, \alpha_{n}\right\}$ is a real $n$-tuple satisfying $\alpha_{1}>\alpha_{2}>\cdots>\alpha_{m}>\gamma>\alpha_{m+1}>$ $\cdots>\alpha_{n}>0(n>m \geq 1), i=1,2, \ldots, n$.

This paper is organized as follows. In the next section, we give some preliminaries and lemmas. In Sections 3 and 4, we will use the Riccati transformation technique to prove our main results. In Section 5, we present two examples to illustrate our results.

\section{Preliminaries and Lemmas}

For convenience, we recall some concepts related to time scales. More details can be found in [9].

Definition 1. Let $\mathbb{T}$ be a time scale; for $t \in \mathbb{T}$ the forward jump operator is defined by $\sigma(t):=\inf \{s \in \mathbb{T}: s>t\}$, the backward jump operator by $\rho(t):=\sup \{s \in \mathbb{T}: s<t\}$, and the graininess function by $\mu(t):=\sigma(t)-t$, where inf $\emptyset:=$ $\sup \mathbb{\mathbb { V }}$ and $\sup \emptyset:=\inf \mathbb{T}$. If $\sigma(t)>t, t$ is said to be right scattered; otherwise, it is right dense. If $\rho(t)<t, t$ is said to be left scattered; otherwise, it is left dense. The set $\mathbb{T}^{\mathcal{K}}$ is defined as follows. If $\mathbb{T}$ has a left-scattered maximum $m$, then $\mathbb{T}^{\kappa}=\mathbb{T}-\{m\}$; otherwise, $\mathbb{T}^{\mathcal{K}}=\mathbb{T}$.

Definition 2. For a function $f: \mathbb{T} \rightarrow \mathbb{R}$ and $t \in \mathbb{T}^{\kappa}$, we define the delta-derivative $f^{\Delta}(t)$ of $f(t)$ to be the number (provided that it exists) with the property that, given any $\varepsilon>0$, there is a neighborhood $U$ of $t$ (i.e., $U=(t-\delta, t+\delta) \cap \mathbb{V}$ for some $\delta$ ) such that

$$
\begin{aligned}
& \left|[f(\sigma(t))-f(s)]-f^{\Delta}(t)[\sigma(t)-s]\right| \\
& \quad \leq \varepsilon|\sigma(t)-s| \quad \forall s \in U .
\end{aligned}
$$

We say that $f$ is delta differentiable (or in short: differentiable) on $\mathbb{T}^{\mathcal{K}}$ provided that $f^{\Delta}(t)$ exists for all $t \in \mathbb{T}^{\kappa}$.

It is easily seen that if $f$ is continuous at $t \in \mathbb{T}$ and $t$ is right scattered, and then $f$ is differentiable at $t$ with

$$
f^{\Delta}(t)=\frac{f(\sigma(t))-f(t)}{\mu(t)} .
$$

Moreover, if $t$ is right dense, then $f$ is differential at $t$ iff the limit

$$
\lim _{s \rightarrow t} \frac{f(t)-f(s)}{t-s}
$$

exists as a finite number. In this case

$$
f^{\Delta}(t)=\lim _{s \rightarrow t} \frac{f(t)-f(s)}{t-s} .
$$

In addition, if $f^{\Delta} \geq 0$, then $f$ is nondecreasing. A useful formula is as follows:

$$
f^{\sigma}(t)=f(t)+\mu(t) f^{\Delta}(t), \quad \text { where } f^{\sigma}(t):=f(\sigma(t)) .
$$

We will make use of the following product and quotient rules for the derivative of the product $f g$ and the quotient $f / g$ (where $g g^{\sigma} \neq 0$ ) of two differentiable functions $f$ and $g$ :

$$
\begin{gathered}
(f g)^{\Delta}=f^{\Delta} g+f^{\sigma} g^{\Delta}=f g^{\Delta}+f^{\Delta} g^{\sigma}, \\
\left(\frac{f}{g}\right)^{\Delta}=\frac{f^{\Delta} g-f g^{\Delta}}{g g^{\sigma}} .
\end{gathered}
$$

Definition 3. Let $f: \mathbb{T} \rightarrow \mathbb{R}$ be a function, $f$ is called right dense continuous (rd-continuous) if it is continuous at 
right-dense points in $\mathbb{T}$, and its left-sided limits exist (finite) at left-dense points in $\mathbb{T}$. A function $F: \mathbb{T} \rightarrow \mathbb{R}$ is called an antiderivative of $f$ provided that $F^{\Delta}(t)=f(t)$ holds for all $t \in \mathbb{T}^{k}$. By the antiderivative, the Cauchy integral of $f$ is defined as $\int_{a}^{b} f(s) \Delta s=F(b)-F(a)$, and $\int_{a}^{\infty} f(s) \Delta s=$ $\lim _{t \rightarrow \infty} \int_{a}^{t} f(s) \Delta s$.

In (9), we assume that $\mathbb{T}$ is a time scale satisfying inf $\mathbb{T}=$ $t_{0}$ and $\sup \mathbb{\mathbb { V }}=\infty$, and

$\left(h_{1}\right) \delta_{i}(t) \in C_{\mathrm{rd}}(\mathbb{T}, \mathbb{T}), \delta_{i}^{\Delta}(t)>0$ and $\lim _{t \rightarrow \infty} \delta_{i}(t)=\infty$, $i=0,1, \ldots, n$;

$\left(h_{2}\right) r(t) \in C_{\mathrm{rd}}(\mathbb{T},(0, \infty))$, and $q_{i}(t), e(t) \in C_{\mathrm{rd}}(\mathbb{T}, \mathbb{R}), i=$ $0,1, \ldots, n$.

By a solution of (9), we mean a nontrivial real-valued function $x(t) \in C_{\mathrm{rd}}^{1}\left[t_{0}, \infty\right)$ satisfying $r(t)\left(x^{\Delta}(t)\right)^{\gamma} \in$ $C_{\text {rd }}^{1}\left[t_{0}, \infty\right)$ and (9). Our attention is restricted to those solutions of (9) that exist on some half-line $\left[t_{x}, \infty\right)$ and satisfy $\sup \left\{|x(t)|: t \geq t_{0}\right\}>0$ for any $t_{0} \geq t_{x}$. A solution $x(t)$ of (9) is said to be oscillatory if it is neither eventually positive nor eventually negative. Otherwise it is called nonoscillatory. The equation itself is called oscillatory if all its solutions are oscillatory.

For convenience, we use the notations $x(\sigma(t))=x^{\sigma}(t)$, $x\left(\delta_{i}(t)\right)=x^{\delta_{i}}(t)(i=1,2, \ldots, n), x^{\Delta}(\sigma(t))=\left(x^{\Delta}(t)\right)^{\sigma}$, and, for $i=0,1,2, \ldots, n$ and $k=1,2$,

$$
\begin{gathered}
\xi_{i, k}(t)=\frac{\int_{\delta_{i}\left(a_{k}\right)}^{\delta_{i}(t)} r^{-1 / \gamma}(s) \Delta s}{\int_{\delta_{i}\left(a_{k}\right)}^{\sigma(t)} r^{-1 / \gamma}(s) \Delta s}, \\
\theta_{i, k}(t)=\frac{\int_{\delta_{i}(t)}^{\delta_{i}\left(b_{k}\right)} r^{-1 / \gamma}(s) \Delta s}{\int_{\sigma(t)}^{\delta_{i}\left(b_{k}\right)} r^{-1 / \gamma}(s) \Delta s}, \\
g_{i, k}(t)= \begin{cases}\xi_{i, k}, & \delta_{i}(t)<\sigma(t), \\
1, & \delta_{i}(t)=\sigma(t), \\
\theta_{i, k}, & \delta_{i}(t)>\sigma(t) .\end{cases}
\end{gathered}
$$

Now, we give the first lemma.

Lemma 4. Let conditions $\left(h_{1}\right)$ and $\left(h_{2}\right)$ hold. Furthermore, assume that, for any $T \geq t_{0}$, there exist constants $a_{k}, b_{k} \in$ $[T, \infty)_{\mathbb{T}}\left(a_{k}<b_{k}, k=1,2\right)$ such that

$$
\begin{gathered}
q_{i}(t) \geq 0 \quad \text { for } t \in\left[G_{1}\left(a_{1}\right), G_{2}\left(b_{1}\right)\right] \cup\left[G_{1}\left(a_{2}\right), G_{2}\left(b_{2}\right)\right], \\
(-1)^{k} e(t) \geq 0 \text { for } t \in\left[G_{1}\left(a_{k}\right), G_{2}\left(b_{k}\right)\right],
\end{gathered}
$$

where $G_{1}(t)=\min \left\{t, \delta_{i}(t)\right\}, G_{2}(t)=\max \left\{t, \delta_{i}(t)\right\}, i=0,1$, $\ldots, n$.

If $x(t)$ is a nonoscillatory solution of (9), then, for $t \in$ $\left[a_{k}, b_{k}\right]$, we have $x\left(\delta_{i}(t)\right) \geq g_{i, k}(t) x(\sigma(t)), i=0,1,2, \ldots, n$, $k=1,2$.
Proof. If $x(t)$ is an eventually positive solution of (9), then by $\left(h_{1}\right)$ there exists a $T \in\left[t_{0}, \infty\right)_{\mathbb{\mathbb { V }}}$ such that

$$
x(t)>0, \quad x\left(\delta_{i}(t)\right)>0 \quad \text { for } t \geq T, i=0,1,2, \ldots, n .
$$

By assumption, we can choose $b_{1}>a_{1}>T$ such that $q_{i}(t) \geq 0$ and $e(t) \leq 0$ on $\left[G_{1}\left(a_{1}\right), G_{2}\left(b_{1}\right)\right]$. By (9) we have

$$
\left(r(t)\left(x^{\Delta}(t)\right)^{\gamma}\right)^{\Delta} \leq 0 \quad \text { for } t \in\left[G_{1}\left(a_{1}\right), G_{2}\left(b_{1}\right)\right],
$$

which implies that $r(t)\left(x^{\Delta}(t)\right)^{\gamma}$ is decreasing on $\left[a_{1}, b_{1}\right] \subset$ $\left[G_{1}\left(a_{1}\right), G_{2}\left(b_{1}\right)\right]$.

Case 1. $t \in\left[a_{1}, b_{1}\right]$ and $\delta_{i}(t)<\sigma(t)$. In this case, we get

$$
\begin{aligned}
x^{\sigma}(t)-x^{\delta_{i}}(t) & =\int_{\delta_{i}(t)}^{\sigma(t)} \frac{\left(r(s)\left(x^{\Delta}(s)\right)^{\gamma}\right)^{1 / \gamma}}{r^{1 / \gamma}(s)} \Delta s \\
& \leq\left(r\left(\delta_{i}(t)\right)\left(x^{\Delta}\left(\delta_{i}(t)\right)\right)^{\gamma}\right)^{1 / \gamma} \int_{\delta_{i}(t)}^{\sigma(t)} \frac{\Delta s}{r^{1 / \gamma}(s)} .
\end{aligned}
$$

It follows that

$$
\frac{x^{\sigma}(t)}{x^{\delta_{i}}(t)}<1+\frac{\left(r\left(\delta_{i}(t)\right)\left(x^{\Delta}\left(\delta_{i}(t)\right)\right)^{\gamma}\right)^{1 / \gamma}}{x^{\delta_{i}}(t)} \int_{\delta_{i}(t)}^{\sigma(t)} \frac{\Delta s}{r^{1 / \gamma}(s)} .
$$

Also, since $\delta_{i}^{\Delta}(t) \geq 0$, we see $\delta_{i}(t) \geq \delta_{i}\left(a_{1}\right)$ for $t \in\left[a_{1}, b_{1}\right]$. So, we get

$$
\begin{aligned}
x^{\delta_{i}}(t) & >x^{\delta_{i}}(t)-x\left(\delta_{i}\left(a_{1}\right)\right) \\
& =\int_{\delta_{i}\left(a_{1}\right)}^{\delta_{i}(t)} \frac{\left(r(s)\left(x^{\Delta}(s)\right)^{\gamma}\right)^{1 / \gamma}}{r^{1 / \gamma}(s)} \Delta s \\
& \geq\left(r\left(\delta_{i}(t)\right)\left(x^{\Delta}\left(\delta_{i}(t)\right)\right)^{\gamma}\right)^{1 / \gamma} \int_{\delta_{i}\left(a_{1}\right)}^{\delta_{i}(t)} \frac{\Delta s}{r^{1 / \gamma}(s)},
\end{aligned}
$$

which implies that

$$
\frac{\left(r\left(\delta_{i}(t)\right)\left(x^{\Delta}\left(\delta_{i}(t)\right)\right)^{\gamma}\right)^{1 / \gamma}}{x^{\delta_{i}}(t)}<\frac{1}{\int_{\delta_{i}\left(a_{1}\right)}^{\delta_{i}(t)}\left(\Delta s / r^{1 / \gamma}(s)\right)} .
$$

It follows that

$$
\begin{aligned}
\frac{x^{\sigma}(t)}{x^{\delta_{i}}(t)} & <1+\frac{\int_{\delta_{i}(t)}^{\sigma(t)}\left(\Delta s / r^{1 / \gamma}(s)\right)}{\int_{\delta_{i}\left(a_{1}\right)}^{\delta_{i}(t)}\left(\Delta s / r^{1 / \gamma}(s)\right)} \\
& \leq \frac{\int_{\delta_{i}\left(a_{1}\right)}^{\sigma(t)}\left(\Delta s / r^{1 / \gamma}(s)\right)}{\int_{\delta_{i}\left(a_{1}\right)}^{\delta_{i}(t)}\left(\Delta s / r^{1 / \gamma}(s)\right)}=\frac{1}{\xi_{i, 1}(t)} .
\end{aligned}
$$


Case 2. $t \in\left[a_{1}, b_{1}\right]$ and $\delta_{i}(t)>\sigma(t)$. In this case, we obtain

$$
\begin{aligned}
x^{\delta_{i}}(t)-x^{\sigma}(t) & =\int_{\sigma(t)}^{\delta_{i}(t)} \frac{\left(r(s)\left(x^{\Delta}(s)\right)^{\gamma}\right)^{1 / \gamma}}{r^{1 / \gamma}(s)} \Delta s \\
& \geq\left(r\left(\delta_{i}(t)\right)\left(x^{\Delta}\left(\delta_{i}(t)\right)\right)^{\gamma}\right)^{1 / \gamma} \int_{\sigma(t)}^{\delta_{i}(t)} \frac{\Delta s}{r^{1 / \gamma}(s)} .
\end{aligned}
$$

Therefore,

$$
\frac{x^{\sigma}(t)}{x^{\delta_{i}}(t)}<1-\frac{\left(r\left(\delta_{i}(t)\right)\left(x^{\Delta}\left(\delta_{i}(t)\right)\right)^{\gamma}\right)^{1 / \gamma}}{x^{\delta_{i}}(t)} \int_{\sigma(t)}^{\delta_{i}(t)} \frac{\Delta s}{r^{1 / \gamma}(s)} .
$$

Also, since $\delta^{\Delta}(t) \geq 0$, we see $\delta_{i}(t) \leq \delta_{i}\left(b_{1}\right)$ for $t \in\left[a_{1}, b_{1}\right]$, and we have

$$
\begin{aligned}
- & x^{\delta_{i}}(t)<x\left(\delta_{i}\left(b_{1}\right)\right)-x\left(\delta_{i}(t)\right) \\
& =\int_{\delta_{i}(t)}^{\delta_{i}\left(b_{1}\right)} \frac{\left(r(s)\left(x^{\Delta}(s)\right)^{\gamma}\right)^{1 / \gamma}}{r^{1 / \gamma}(s)} \Delta s \\
& \leq\left(r\left(\delta_{i}(t)\right)\left(x^{\Delta}\left(\delta_{i}(t)\right)\right)^{\gamma}\right)^{1 / \gamma} \int_{\delta_{i}(t)}^{\delta_{i}\left(b_{1}\right)} \frac{\Delta s}{r^{1 / \gamma}(s)},
\end{aligned}
$$

which implies that

$$
-\frac{\left(r\left(\delta_{i}(t)\right)\left(x^{\Delta}\left(\delta_{i}(t)\right)\right)^{\gamma}\right)^{1 / \gamma}}{x^{\delta_{i}}(t)}<\frac{1}{\int_{\delta_{i}(t)}^{\delta_{i}\left(b_{1}\right)}\left(\Delta s / r^{1 / \gamma}(s)\right)} .
$$

It follows that

$$
\begin{aligned}
\frac{x^{\sigma}(t)}{x^{\delta_{i}}(t)} & <1+\frac{\int_{\sigma(t)}^{\delta_{i}(t)}\left(\Delta s / r^{1 / \gamma}(s)\right)}{\int_{\delta_{i}(t)}^{\delta_{t}\left(b_{1}\right)}\left(\Delta s / r^{1 / \gamma}(s)\right)} \\
& \leq \frac{\int_{\sigma(t)}^{\delta_{i}\left(b_{1}\right)}\left(\Delta s / r^{1 / \gamma}(s)\right)}{\int_{\delta_{i}(t)}^{\delta_{i}\left(b_{1}\right)}\left(\Delta s / r^{1 / \gamma}(s)\right)}=\frac{1}{\theta_{i, 1}(t)} .
\end{aligned}
$$

Case 3. $t \in\left[a_{1}, b_{1}\right]$ and $\delta_{i}(t)=\sigma(t)$. It is easy to get

$$
\frac{x\left(\delta_{i}(t)\right)}{x(\sigma(t))}=1 .
$$

Combing (24), (29), and (30), we have

$$
x\left(\delta_{i}(t)\right) \geq g_{i, 1}(t) x^{\sigma}(t), \quad i=0,1, \ldots, n, t \in\left[a_{1}, b_{1}\right] .
$$

When $x(t)$ is an eventually negative solution of (9), its proof follows the similar argument using the interval $\left[G_{1}\left(a_{2}\right), G_{2}\left(b_{2}\right)\right]$ instead of $\left[G_{1}\left(a_{1}\right), G_{2}\left(b_{1}\right)\right]$.

The proof is complete.

Lemma 5 (see [2]). Let $\alpha_{i}, i=1,2, \ldots, n$, be the $n$-tuple satisfying $\alpha_{1}>\alpha_{2}>\cdots>\alpha_{m}>\gamma>\alpha_{m+1}>\cdots>\alpha_{n}>0$.
Then there exists an $n$-tuple $\left(\eta_{1}, \ldots, \eta_{n}\right)$ with $0<\eta_{i}<1$ satisfying

$$
\sum_{i=1}^{n} \alpha_{i} \eta_{i}=\gamma
$$

and which also satisfies either

$$
\sum_{i=1}^{n} \eta_{i}<1
$$

or

$$
\sum_{i=1}^{n} \eta_{i}=1
$$

Lemma 6 (see [7]). Let $\alpha, \beta, u, A$, and $B$ be positive real numbers and let $\gamma$ be a quotient of odd positive integers. Then

$$
\begin{aligned}
& A u^{\gamma}-B u^{\gamma-\alpha} \geq-\alpha\left(\left(\frac{\gamma-\alpha}{A}\right)^{\gamma-\alpha}\left(\frac{B}{\gamma}\right)^{\gamma}\right)^{1 / \alpha}, \quad 0<\alpha<\gamma, \\
& A u^{\beta-\gamma}+B u^{-\gamma} \geq \beta\left(\left(\frac{B}{\beta-\gamma}\right)^{\beta-\gamma}\left(\frac{A}{\gamma}\right)^{\gamma}\right)^{1 / \beta}, \quad 0<\gamma<\beta .
\end{aligned}
$$

Lemma 7 (Yong's Inequality). If $p>1$ and $q>1$ are conjugate numbers $(1 / p+1 / q=1)$, then

$$
\frac{|u|^{p}}{p}+\frac{|v|^{q}}{q} \geq|u v|, \quad u v \in R
$$

and equality holds if and only if $u=|v|^{q-2} v$.

Let $\beta>\alpha, u=A^{\alpha / \beta} x^{\alpha}, p=\beta / \alpha$, and $v=(B \alpha)^{1-(\alpha / \beta)}(\beta-$ $\alpha)^{(\alpha / \beta)-1}$. It follows from Lemma 7 that

$$
A x^{\beta}+B \geq \beta \alpha^{-\alpha / \beta}(\beta-\alpha)^{(\alpha / \beta)-1} A^{\alpha / \beta} B^{1-(\alpha / \beta)} x^{\alpha}
$$

for all $A, B, x \geq 0$. Rewriting the above inequality we also have

$$
C x^{\alpha}-D \leq \alpha \beta^{-\beta / \alpha}(\beta-\alpha)^{(\beta / \alpha)-1} C^{\beta / \alpha} D^{1-(\beta / \alpha)} x^{\beta}
$$

for all $C, D, x \geq 0$.

\section{Main Results}

In this section, by employing the Riccati transformation technique we will establish oscillation criteria for (9). Set

$$
Q_{1, k}(t)=q_{0}(t) g_{0, k}^{\gamma}(t)+\left(\eta_{0}^{-1}|e(t)|\right)^{\eta_{0}} \prod_{i=1}^{n}\left(\eta_{i}^{-1} q_{i}(t) g_{i, k}^{\alpha_{i}}(t)\right)^{\eta_{i}},
$$

$$
Q_{2, k}(t)=q_{0}(t) g_{0, k}^{\gamma}(t)+\prod_{i=1}^{n}\left(\eta_{i}^{-1} q_{i}(t) g_{i, k}^{\alpha_{i}}(t)\right)^{\eta_{i}}
$$




$$
\begin{aligned}
Q_{3, k}(t)= & q_{0}(t) g_{0, k}^{\gamma}(t) \\
& +\sum_{i=1}^{m} \alpha_{i}\left(\left(\frac{q_{i}(t) g_{i, k}^{\alpha_{i}}(t)}{\gamma}\right)^{\gamma}\left(\frac{\lambda_{i}|e(t)|}{\alpha_{i}-\gamma}\right)^{\alpha_{i}-\gamma}\right)^{1 / \alpha_{i}} \\
& -\sum_{i=m+1}^{n} \alpha_{i}\left(\left(\frac{\left(q_{i}(t)\right)_{-} g_{i, k}^{\alpha_{i}}(t)}{\gamma}\right)^{\gamma}\left(\frac{\gamma-\alpha_{i}}{\lambda_{i}|e(t)|}\right)^{\gamma-\alpha_{i}}\right)^{1 / \alpha_{i}} .
\end{aligned}
$$

We define for $a, b \in\left[t_{0}, \infty\right)_{\mathbb{W}}$ with $a<b$ the admissible set

$$
A(a, b):=\left\{u \in C_{\mathrm{rd}}^{1}[a, b]: u(a)=u(b)=0, u \neq \equiv\right\} .
$$

By employing (32) and (33) in Lemma 5, we have the following theorem.

Theorem 8. Let conditions $\left(h_{1}\right)$ and $\left(h_{2}\right)$ hold. Furthermore, assume that, for any $T \geq t_{0}$, there exist constants $a_{k}, b_{k} \in$ $[T, \infty)_{\mathbb{T}}\left(a_{k}<b_{k}, k=1,2\right)$ such that (17) holds. If there exists a function $u \in A\left(a_{k}, b_{k}\right)$ such that

$$
\int_{a_{k}}^{b_{k}}\left[\left(u^{\sigma}(s)\right)^{\gamma+1} Q_{1, k}(s)-\left(u^{\Delta}(s)\right)^{\gamma+1} r(s)\right] \Delta s \geq 0, \quad k=1,2,
$$

then (9) is oscillatory, where $\eta_{i}>0(i=1,2, \ldots, n)$ satisfy (32)-(33) and $\eta_{0}=1-\sum_{i=1}^{n} \eta_{i}$.

Proof. Suppose to the contrary that $x(t)$ is a nonoscillatory solution of (9). Without loss of generality, we may assume that $x(t)$ is eventually positive. Then, there exists $T \geq t_{0}$ sufficiently large such that $x(t)>0, x\left(\delta_{i}(t)\right)>0 i=$ $0,1,2, \ldots, n$, for all $t \geq T$. By assumption, we can choose $b_{1}>a_{1}>T$, such that $q_{i}(t) \geq 0$ and $e(t) \leq 0$ on the interval $\left[G_{1}\left(a_{1}\right), G_{2}\left(b_{1}\right)\right]$. From Lemma 4 and (9), we obtain, for $t \in\left[a_{1}, b_{1}\right]$,

$$
\left(r(t)\left(x^{\Delta}(t)\right)^{\gamma}\right)^{\Delta}+\sum_{i=0}^{n} q_{i}(t) g_{i, 1}^{\alpha_{i}}(t) x^{\alpha_{i}}(\sigma(t)) \leq e(t) .
$$

Defining the function $w(t)$ by the Riccati substitution

$$
w(t):=\frac{-r(t)\left(x^{\Delta}(t)\right)^{\gamma}}{x^{\gamma}(t)} \text { for } t \in\left[a_{1}, b_{1}\right]
$$

and then

$$
w^{\Delta}(t)=-\frac{\left(r(t)\left(x^{\Delta}(t)\right)^{\gamma}\right)^{\Delta}}{x^{\gamma}(\sigma(t))}-\left(r(t)\left(x^{\Delta}(t)\right)^{\gamma}\right)\left(\frac{1}{x^{\gamma}(t)}\right)^{\Delta} .
$$

By (43), we obtain

$$
\begin{aligned}
& \frac{\left(r(t)\left(x^{\Delta}(t)\right)^{\gamma}\right)^{\Delta}}{x^{\gamma}(\sigma(t))} \\
& \leq-\sum_{i=0}^{n} q_{i}(t) g_{i, 1}^{\alpha_{i}}(t) x^{\alpha_{i}-\gamma}(\sigma(t))+e(t) x^{-\gamma}(\sigma(t)) \\
& =-q_{0}(t) g_{0,1}^{\gamma}(t) \\
& \quad-\sum_{i=1}^{n} q_{i}(t) g_{i, 1}^{\alpha_{i}}(t) x^{\alpha_{i}-\gamma}(\sigma(t))-|e(t)| x^{-\gamma}(\sigma(t)) .
\end{aligned}
$$

Corresponding to the exponents $\alpha_{i}(i=1,2, \ldots, n)$ in (9), let $\eta_{i}(i=1,2, \ldots, n)$ be chosen to satisfy (32) and (33) in Lemma 5 and set $\eta_{0}=1-\sum_{i=1}^{n} \eta_{i}$.

Setting

$$
a_{0}=\eta_{0}^{-1}|e(t)|, \quad a_{i}=\eta_{i}^{-1} q_{i}(t) g_{i, 1}^{\alpha_{i}}(t) x^{\alpha_{i}}(\sigma(t))
$$

and by the arithmetic geometric mean inequality in [21],

$$
\sum_{i=0}^{n} \eta_{i} a_{i} \geq \prod_{i=0}^{n} a_{i}^{\eta_{i}}, \quad a_{i} \geq 0
$$

we get for $t \in\left[a_{1}, b_{1}\right]$

$$
\begin{aligned}
\sum_{i=1}^{n} q_{i}(t) g_{i, 1}^{\alpha_{i}}(t) x^{\alpha_{i}-\gamma}(\sigma(t))+|e(t)| x^{-\gamma}(\sigma(t)) & \\
= & x^{-\gamma}(\sigma(t))\left[\left(\eta_{0} \eta_{0}^{-1}|e(t)|\right)\right. \\
& \left.+\sum_{i=1}^{n} \eta_{i} \eta_{i}^{-1} q_{i}(t) g_{i, 1}^{\alpha_{i}}(t) x^{\alpha_{i}}(\sigma(t))\right] \\
\geq & x^{-\gamma}(\sigma(t))\left(\eta_{0}^{-1}|e(t)|\right)^{\eta_{0}} \\
& \times \prod_{i=1}^{n}\left(\eta_{i}^{-1} q_{i}(t) g_{i, 1}^{\alpha_{i}}(t) x^{\alpha_{i}}(\sigma(t))\right)^{\eta_{i}} \\
= & x^{-\gamma}(\sigma(t))\left(\eta_{0}^{-1}|e(t)|\right)^{\eta_{0}} \\
& \times \prod_{i=1}^{n}\left(\eta_{i}^{-1} q_{i}(t) g_{i, 1}^{\alpha_{i}}(t)\right)^{\eta_{i}}(x(\sigma(t)))^{\sum_{i=1}^{n} \alpha_{i} \eta_{i} .} .
\end{aligned}
$$

For (32), we have

$$
\begin{aligned}
& \frac{\left(r(t)\left(x^{\Delta}(t)\right)^{\gamma}\right)^{\Delta}}{x^{\gamma}(\sigma(t))} \\
& \leq-q_{0}(t) g_{0,1}^{\gamma}(t)-\left(\eta_{0}^{-1}|e(t)|\right)^{\eta_{0}} \\
& \quad \times \prod_{i=1}^{n}\left(\eta_{i}^{-1} q_{i}(t) g_{i, 1}^{\alpha_{i}}(t)\right)^{\eta_{i}} .
\end{aligned}
$$


By (45)-(50), we get

$$
w^{\Delta}(t)>Q_{1,1}(t)+\frac{r(t)\left(x^{\Delta}(t)\right)^{\gamma}\left(x^{\gamma}(t)\right)^{\Delta}}{x^{\gamma}(t) x^{\gamma}(\sigma(t))} .
$$

Set $\Phi_{\gamma}(y)=|y|^{\gamma-1} y$. Since $\gamma>0$ is a quotient of odd positive integers, it is easy to prove $\Phi_{\gamma}(y)=|y|^{\gamma-1} y=y^{\gamma}$. Multiplying by $\left(u^{\sigma}(t)\right)^{\gamma+1}$ on (51) and then using the identity

$$
\left(u^{\gamma+1}(t) w(t)\right)^{\Delta}=\left(u^{\sigma}(t)\right)^{\gamma+1} w^{\Delta}(t)+\left(u^{\gamma+1}(t)\right)^{\Delta} w(t),
$$

we obtain

$$
\begin{aligned}
\left(u^{\gamma+1}(t) w(t)\right)^{\Delta}> & \left(u^{\sigma}(t)\right)^{\gamma+1} Q_{1,1}(t)+\left(u^{\gamma+1}(t)\right)^{\Delta} w(t) \\
& +\left(u^{\sigma}(t)\right)^{\gamma+1} \frac{r(t)\left(x^{\Delta}(t)\right)^{\gamma}\left(x^{\gamma}(t)\right)^{\Delta}}{x^{\gamma}(t) x^{\gamma}(\sigma(t))} \\
= & \left(u^{\sigma}(t)\right)^{\gamma+1} Q_{1,1}(t)-\left(u^{\Delta}(t)\right)^{\gamma+1} r(t) \\
& +G(u, w),
\end{aligned}
$$

where

$$
\begin{aligned}
G(u, w)= & \left(u^{\Delta}(t)\right)^{\gamma+1} r(t)+\left(u^{\gamma+1}(t)\right)^{\Delta} w(t) \\
& +\left(u^{\sigma}(t)\right)^{\gamma+1} \frac{r(t)\left(x^{\Delta}(t)\right)^{\gamma}\left(x^{\gamma}(t)\right)^{\Delta}}{x^{\gamma}(t) x^{\gamma}(\sigma(t))} \\
= & \left(u^{\Delta}(t)\right)^{\gamma+1} r(t)+\left(u^{\gamma+1}(t)\right)^{\Delta} w(t) \\
& +\left(u^{\sigma}(t)\right)^{\gamma+1} \frac{r(t) \Phi_{\gamma}\left(x^{\Delta}(t)\right)\left(\Phi_{\gamma}(x(t))\right)^{\Delta}}{\Phi_{\gamma}(x(t)) \Phi_{\gamma}(x(\sigma(t)))} .
\end{aligned}
$$

As demonstrated in [10], we know that $G(u, w) \geq 0$ and that $G(u, w)=0$ if and only if

$$
\Phi_{\gamma}^{-1}\left(-\frac{w(t)}{r(t)}\right) u(t)=u^{\Delta}(t)
$$

where $\Phi_{\gamma}^{-1}$ stands for the inverse function. In our case, sine $1+\mu \Phi_{\gamma}^{-1}(-w(t) / r(t))=x^{\sigma}(t) / x(t)>0$, and dynamic equation (55) has a unique solution satisfying $u\left(a_{1}\right)=0$. Clearly, the unique solution is $u \equiv 0$. Therefore, $G(u, w)>0$ on $\left[a_{1}, b_{1}\right]$. So, we get

$$
\left(u^{\gamma+1}(t) w(t)\right)^{\Delta}>\left(u^{\sigma}(t)\right)^{\gamma+1} Q_{1,1}(t)-\left(u^{\Delta}(t)\right)^{\gamma+1} r(t) .
$$

Integrating from $a_{1}$ to $b_{1}$ and using $u\left(a_{1}\right)=u\left(b_{1}\right)=0$, we find

$$
\int_{a_{1}}^{b_{1}}\left[\left(u^{\sigma}(s)\right)^{\gamma+1} Q_{1,1}(s)-\left(u^{\Delta}(s)\right)^{\gamma+1} r(s)\right] \Delta s<0,
$$

which leads to a contradiction to (42).
The proof when $x(t)$ is eventually negative follows the similar arguments using the interval $\left[G_{1}\left(a_{2}\right), G_{2}\left(b_{2}\right)\right]$ instead of $\left[G_{1}\left(a_{1}\right), G_{2}\left(b_{2}\right)\right]$

The proof is complete.

By employing (32) and (34) in Lemma 5, we have the following theorem.

Theorem 9. Let conditions $\left(h_{1}\right)$ and $\left(h_{2}\right)$ hold. Furthermore, assume that, for any $T \geq t_{0}$, there exist constants $a_{k}, b_{k} \in$ $[T, \infty)_{\mathbb{T}}\left(a_{k}<b_{k}, k=1,2\right)$ such that $(17)$ holds. If there exists a function $u \in A\left(a_{k}, b_{k}\right)$ such that

$\int_{a_{k}}^{b_{k}}\left[\left(u^{\sigma}(s)\right)^{\gamma+1} Q_{2, k}(s)-\left(u^{\Delta}(s)\right)^{\gamma+1} r(s)\right] \Delta s \geq 0, \quad k=1,2$,

then (9) is oscillatory, where $\eta_{i}>0(i=1,2, \ldots, n)$ satisfy (32) and (34).

Proof. Suppose to the contrary that $x(t)$ is a nonoscillatory solution of (9). Without loss of generality, we may assume that $x(t)$ is eventually positive. Then, there exists $T \geq t_{0}$ sufficiently large such that $x(t)>0, x\left(\delta_{i}(t)\right)>0, i=$ $0,1,2, \ldots, n$, for all $t \geq T$. By assumption, we can choose $b_{1}>a_{1}>T$, such that $q_{i}(t) \geq 0$ and $e(t) \leq 0$ on the interval $\left[G_{1}\left(a_{1}\right), G_{2}\left(b_{1}\right)\right]$. Define the Riccati substitution $w(t)$ as (44). Let $\eta_{i}(i=1,2, \ldots, n)$ be chosen to satisfy (32) and (34) in Lemma 5. By (46), we can get

$$
\begin{aligned}
& \frac{\left(r(t)\left(x^{\Delta}(t)\right)^{\gamma}\right)^{\Delta}}{x^{\gamma}(\sigma(t))} \\
& \leq-q_{0}(t) g_{0,1}^{\gamma}(t) \\
& \quad-\sum_{i=1}^{n} q_{i}(t) g_{i, 1}^{\alpha_{i}}(t) x^{\alpha_{i}-\gamma}(\sigma(t))-|e(t)| x^{-\gamma}(\sigma(t)) \\
& \leq-q_{0}(t) g_{0,1}^{\gamma}(t)-x^{-\gamma}(\sigma(t)) \\
& \quad \times \sum_{i=1}^{n} \eta_{i} \eta_{i}^{-1} q_{i}(t) g_{i, 1}^{\alpha_{i}}(t) x^{\alpha_{i}}(\sigma(t)) .
\end{aligned}
$$

Setting

$$
a_{i}=\frac{1}{\eta_{i}} q_{i}(t) g_{i, 1}^{\alpha_{i}}(t) x^{\alpha_{i}}(\sigma(t)) \quad(i=1,2, \ldots, n),
$$

using again the arithmetic-geometric mean inequality in [21],

$$
\sum_{i=1}^{n} \eta_{i} a_{i} \geq \prod_{i=1}^{n} a_{i}^{\eta_{i}}, \quad a_{i} \geq 0,
$$

and similar to (50), we have

$$
\frac{\left(r(t)\left(x^{\Delta}(t)\right)^{\gamma}\right)^{\Delta}}{x^{\gamma}(\sigma(t))} \leq-q_{0}(t) g_{0,1}^{\gamma}(t)-\prod_{i=1}^{n}\left(\eta_{i}^{-1} q_{i}(t) g_{i, 1}^{\alpha_{i}}(t)\right)^{\eta_{i}} .
$$


By (45) and (62), we get

$$
w^{\Delta}(t)>Q_{2,1}(t)+\frac{r(t)\left(x^{\Delta}(t)\right)^{\gamma}\left(x^{\gamma}(t)\right)^{\Delta}}{x^{\gamma}(t) x^{\gamma}(\sigma(t))} .
$$

The reminder of the proof is similar to that of Theorem 8 . The proof is complete.

By employing (35) and (36) in Lemma 6, we have the following theorem.

Theorem 10. Let conditions $\left(h_{1}\right)$ and $\left(h_{2}\right)$ hold. Furthermore, assume that, for any $T \geq t_{0}$, there exist constants $a_{k}, b_{k} \in$ $[T, \infty)_{\mathbb{T}}\left(a_{k}<b_{k}, k=1,2\right)$ such that (17) holds. If there exists a function $u \in A\left(a_{k}, b_{k}\right)$ such that

$$
\int_{a_{k}}^{b_{k}}\left[\left(u^{\sigma}(s)\right)^{\gamma+1} Q_{3, k}(s)-\left(u^{\Delta}(s)\right)^{\gamma+1} r(s)\right] \Delta s \geq 0, \quad k=1,2,
$$

then (9) is oscillatory, where $\lambda_{i}(i=1,2, \ldots, n)$ are positive numbers with $\sum_{i=1}^{n} \lambda_{i}=1$ and $\left(q_{i}(t)\right)_{-}=\max \left\{-q_{i}(t), 0\right\}, i=$ $1,2, \ldots, n$.

Proof. Suppose to the contrary that $x(t)$ is a nonoscillatory solution of (9). Without loss of generality, we may assume that $x(t)$ is eventually positive. Then, there exists $T \geq t_{0}$ sufficiently large such that $x(t)>0, x\left(\delta_{i}(t)\right)>0, i=0,1,2$, $\ldots, n$, for all $t \geq T$. By assumption, we can choose $b_{1}>$ $a_{1}>T$, such that $q_{i}(t) \geq 0$ and $e(t) \leq 0$ on the interval $\left[G_{1}\left(a_{1}\right), G_{2}\left(b_{1}\right)\right]$. Define the Riccati substitution $w(t)$ as $(44)$. Let $\lambda_{i}(i=1,2, \ldots, n)$ be chosen to satisfy $\sum_{i=1}^{n} \lambda_{i}=1$. Similar to the proof of Theorem 8 , we can get

$$
\begin{aligned}
& \frac{\left(r(t)\left(x^{\Delta}(t)\right)^{\gamma}\right)^{\Delta}}{x^{\gamma}(\sigma(t))} \\
& \leq-q_{0}(t) g_{0,1}^{\gamma}(t)-\sum_{i=1}^{m}\left[q_{i}(t) g_{i, 1}^{\alpha_{i}}(t) x^{\alpha_{i}-\gamma}(\sigma(t))\right. \\
& \left.\quad+\lambda_{i}|e(t)| x^{-\gamma}(\sigma(t))\right] \\
& \quad-\sum_{i=m+1}^{n}\left[q_{i}(t) g_{i, 1}^{\alpha_{i}}(t) x^{\alpha_{i}-\gamma}(\sigma(t))+\lambda_{i}|e(t)| x^{-\gamma}(\sigma(t))\right] .
\end{aligned}
$$

From (36), we get, for $t \in\left(a_{1}, b_{1}\right)$ and $\alpha_{i}>\gamma(i=1,2, \ldots, m)$,

$$
\begin{gathered}
q_{i}(t) g_{i, 1}^{\alpha_{i}}(t) x^{\alpha_{i}-\gamma}(\sigma(t))+\lambda_{i}|e(t)| x^{-\gamma}(\sigma(t)) \\
\quad \geq \alpha_{i}\left(\left(\frac{q_{i}(t) g_{i, 1}^{\alpha_{i}}(t)}{\gamma}\right)^{\gamma}\left(\frac{\lambda_{i}|e(t)|}{\alpha_{i}-\gamma}\right)^{\alpha_{i}-\gamma}\right)^{1 / \alpha_{i}} .
\end{gathered}
$$

From (35), we get, for $t \in\left(a_{1}, b_{1}\right)$ and $\alpha_{i}<\gamma(i=m+1, m+$ $2, \ldots, n)$,

$$
\begin{aligned}
& q_{i}(t) g_{i, 1}^{\alpha_{i}}(t) x^{\alpha_{i}-\gamma}(\sigma(t))+\lambda_{i}|e(t)| x^{-\gamma}(\sigma(t)) \\
& \geq \lambda_{i}|e(t)| x^{-\gamma}(\sigma(t))-\left(q_{i}(t)\right)_{-} g_{i, 1}^{\alpha_{i}}(t) x^{\alpha_{i}-\gamma}(\sigma(t)) \\
& \quad \geq-\alpha_{i}\left(\left(\frac{\left(q_{i}(t)\right)_{-} g_{i, 1}^{\alpha_{i}}(t)}{\gamma}\right)^{\gamma}\left(\frac{\gamma-\alpha_{i}}{\lambda_{i}|e(t)|}\right)^{\gamma-\alpha_{i}}\right)^{1 / \alpha_{i}} .
\end{aligned}
$$

By (45), (65), (66), and (67), we get

$$
w^{\Delta}(t)>Q_{3,1}(t)+\frac{r(t)\left(x^{\Delta}(t)\right)^{\gamma}\left(x^{\gamma}(t)\right)^{\Delta}}{x^{\gamma}(t) x^{\gamma}(\sigma(t))} .
$$

The reminder of the proof is similar to that of Theorem 8 . The proof is complete.

By employing (38) and (39), we have the following theorem.

Theorem 11. Let conditions $\left(h_{1}\right)$ and $\left(h_{2}\right)$ hold. Furthermore, assume that, for any $T \geq t_{0}$, there exist constants $a_{k}, b_{k} \in$ $[T, \infty)_{\mathbb{T}}\left(a_{k}<b_{k}, k=1,2\right)$ such that $(17)$ holds. If there exists a function $u \in A\left(a_{k}, b_{k}\right)$ such that

$$
\begin{array}{r}
\int_{a_{k}}^{b_{k}}\left[\left(u^{\sigma}(s)\right)^{\gamma+1} Q_{4, k}(s)-\left(u^{\Delta}(s)\right)^{\gamma+1} r(s)\right] \\
\Delta s \geq 0, \\
k=1,2,
\end{array}
$$

then (9) is oscillatory, where $\lambda_{i}(i=1,2, \ldots, n)$ are positive numbers with $\sum_{i=1}^{n} \lambda_{i}=1,\left(q_{i}(t)\right)_{-}=\max \left\{-q_{i}(t), 0\right\}, i=$ $1,2, \ldots, n$,

$$
\begin{aligned}
P_{i, k}(t)= & \alpha_{i} \gamma^{-\gamma / \alpha_{i}}\left(\alpha_{i}-\gamma\right)^{\left(\gamma / \alpha_{i}\right)-1}\left(q_{i}(t) g_{i, k}^{\alpha_{i}}(t)\right)^{\gamma / \alpha_{i}} \\
& \times\left(\lambda_{i}|e(t)|\right)^{1-\left(\gamma / \alpha_{i}\right)}, \\
R_{i, k}(t)= & \alpha_{i} \gamma^{-\gamma / \alpha_{i}}\left(\gamma-\alpha_{i}\right)^{\left(\gamma / \alpha_{i}\right)-1}\left(\left(q_{i}(t)\right)_{-} g_{i, k}^{\alpha_{i}}(t)\right)^{\gamma / \alpha_{i}} \\
& \times\left(\lambda_{i}|e(t)|\right)^{1-\left(\gamma / \alpha_{i}\right)}, \\
Q_{4, k}(t)= & q_{0}(t) g_{0, k}^{\gamma}(t)+\sum_{i=1}^{m} P_{i, k}(t)-\sum_{i=m+1}^{n} R_{i, k}(t) .
\end{aligned}
$$

Proof. Suppose to the contrary that $x(t)$ is a nonoscillatory solution of (9). Without loss of generality, we may assume that $x(t)$ is eventually positive. Then, there exists $T \geq t_{0}$ sufficiently large such that $x(t)>0, x\left(\delta_{i}(t)\right)>0, i=0,1,2$, $\ldots, n$, for all $t \geq T$. By assumption, we can choose $b_{1}>$ $a_{1}>T$, such that $q_{i}(t) \geq 0$ and $e(t) \leq 0$ on the interval 
$\left[G_{1}\left(a_{1}\right), G_{2}\left(b_{1}\right)\right]$. Define the Riccati substitution $w(t)$ as (44). By (43), we have

$$
\begin{aligned}
& \left(r(t)\left(x^{\Delta}(t)\right)^{\gamma}\right)^{\Delta}+q_{0}(t) g_{0,1}^{\gamma}(t) x^{\gamma}(\sigma(t)) \\
& \quad+\sum_{i=1}^{m}\left[q_{i}(t) g_{i, 1}^{\alpha_{i}}(t) x^{\alpha_{i}}(\sigma(t))+\lambda_{i}|e(t)|\right] \\
& \quad-\sum_{i=m+1}^{n}\left[-q_{i}(t) g_{i, 1}^{\alpha_{i}}(t) x^{\alpha_{i}}(\sigma(t))-\lambda_{i}|e(t)|\right] \leq 0 .
\end{aligned}
$$

Applying (38) and setting

$$
\begin{gathered}
A=q_{i}(t) g_{i, 1}^{\alpha_{i}}(t), \quad B=\lambda_{i}|e(t)|, \\
\beta=\alpha_{i}, \quad \alpha=\gamma\left(\alpha_{i}>\gamma\right),
\end{gathered}
$$

we have

$$
\sum_{i=1}^{m}\left[q_{i}(t) g_{i, 1}^{\alpha_{i}}(t) x^{\alpha_{i}}(\sigma(t))+\lambda_{i}|e(t)|\right] \geq \sum_{i=1}^{m} P_{i, 1}(t) x^{\gamma}(\sigma(t)) .
$$

On the other hand, we can get

$$
\begin{aligned}
& \sum_{i=m+1}^{n}\left[-q_{i}(t) g_{i, 1}^{\alpha_{i}}(\sigma(t)) x^{\alpha_{i}}(t)-\lambda_{i}|e(t)|\right] \\
& \quad \leq \sum_{i=m+1}^{n}\left[\left(q_{i}(t)\right)_{-} g_{i, 1}^{\alpha_{i}}(t) x^{\alpha_{i}}(\sigma(t))-\lambda_{i}|e(t)|\right] .
\end{aligned}
$$

Applying (39) and setting

$$
\begin{gathered}
C=\left(q_{i}(t)\right)_{-} g_{i, 1}^{\alpha_{i}}(t), \quad D=\lambda_{i}|e(t)|, \\
\beta=\gamma, \quad \alpha=\alpha_{i}\left(\gamma>\alpha_{i}\right),
\end{gathered}
$$

we obtain that

$$
\begin{aligned}
& \sum_{i=m+1}^{n}\left[\left(q_{i}(t)\right)_{-} g_{i, 1}^{\alpha_{i}}(t) x^{\alpha_{i}}(\sigma(t))+\lambda_{i}|e(t)|\right] \\
& \quad \leq \sum_{i=m+1}^{n} R_{i, 1}(t) x^{\gamma}(\sigma(t)) .
\end{aligned}
$$

From (71), (73), and (76), we have

$$
\begin{aligned}
\left(r(t)\left(x^{\Delta}(t)\right)^{\gamma}\right)^{\Delta} \leq & -q_{0}(t) g_{0,1}^{\gamma}(t) x^{\gamma}(\sigma(t)) \\
& -\sum_{i=1}^{m} P_{i, 1}(t) x^{\gamma}(\sigma(t)) \\
& +\sum_{i=m+1}^{n} R_{i, 1}(t) x^{\gamma}(\sigma(t)) .
\end{aligned}
$$

By (45) and (77), we have

$$
w^{\Delta}(t)>Q_{4,1}(t)+\frac{r(t)\left(x^{\Delta}(t)\right)^{\gamma}\left(x^{\gamma}(t)\right)^{\Delta}}{x^{\gamma}(t) x^{\gamma}(\sigma(t))} .
$$

The reminder of the proof is similar to that of Theorem 8 . The proof is complete.

\section{Forms Related to (9)}

Related to (9) are the dynamic equations with mixed delta and nabla derivatives:

$$
\begin{aligned}
& \left(r(t)\left(x^{\Delta}(t)\right)^{\gamma}\right)^{\nabla}+\sum_{i=0}^{n} q_{i}(t)\left|x\left(\delta_{i}(t)\right)\right|^{\alpha_{i}} \operatorname{sgn} x\left(\delta_{i}(t)\right)=e(t), \\
& \left(r(t)\left(x^{\nabla}(t)\right)^{\gamma}\right)^{\Delta}+\sum_{i=0}^{n} q_{i}(t)\left|x\left(\delta_{i}(t)\right)\right|^{\alpha_{i}} \operatorname{sgn} x\left(\delta_{i}(t)\right)=e(t) .
\end{aligned}
$$

It is not difficult to see that time scale modifications of the previous arguments give rise to completely parallel results for the above dynamic equations. For an illustrative example we provide below the version of Theorem 8 for (79), for which a lemma analogous to Lemma 4 can be stated easily. The other theorems for (79) and (80) can be obtained by employing arguments developed for (9).

Set

$$
\begin{gathered}
\zeta_{i, k}(t)=\frac{\int_{\delta_{i}\left(a_{k}\right)}^{\delta_{i}(t)} r^{-1 / \gamma}(s) \Delta s}{\int_{\delta_{i}\left(a_{k}\right)}^{\rho(t)} r^{-1 / \gamma}(s) \Delta s}, \\
\eta_{i, k}(t)=\frac{\int_{\delta_{i}(t)}^{\delta_{i}\left(b_{k}\right)} r^{-1 / \gamma}(s) \Delta s}{\int_{\rho(t)}^{\delta_{i}\left(b_{k}\right)} r^{-1 / \gamma}(s) \Delta s}, \\
p_{i, k}(t)= \begin{cases}\zeta_{i, k}, & \delta_{i}(t)<\rho(t), \\
1, & \delta_{i}(t)=\rho(t), \\
\eta_{i, k}, & \delta_{i}(t)>\rho(t),\end{cases} \\
\times \prod_{5, k}(t)=q_{0}(t) p_{0, k}^{\gamma}(t)+\left(\eta_{0}^{-1}|e(t)|\right)^{\eta_{0}}
\end{gathered}
$$

Theorem 12. Let conditions $\left(h_{1}\right)$ and $\left(h_{2}\right)$ hold. Furthermore, assume that, for any $T \geq t_{0}$, there exist constants $a_{k}, b_{k} \in$ $[T, \infty)_{\mathbb{T}}\left(a_{k}<b_{k}, k=1,2\right)$ such that $(17)$ holds. If there exists a function $u \in A_{*}\left(a_{k}, b_{k}\right):=\left\{u \in C_{\mathrm{ld}}^{1}\left[a_{k}, b_{k}\right]: u\left(a_{k}\right)=u\left(b_{k}\right)=\right.$ $0, u(t) \neq \equiv 0\}$ such that

$$
\int_{a_{k}}^{b_{k}}\left[\left(u^{\rho}(s)\right)^{\gamma+1} Q_{5, k}(s)-\left(u^{\nabla}(s)\right)^{\gamma+1} r(s)\right] \nabla s \geq 0, \quad k=1,2,
$$

then (79) is oscillatory, where $\rho$ denotes the backward jump operator, and $\eta_{i}>0(i=1,2, \ldots, n)$ satisfy (32)-(33) and $\eta_{0}=1-\sum_{i=1}^{n} \eta_{i}$.

\section{Examples}

In this section, we give two examples to illustrate our main results. We first give an example to show Theorems 8 . 
Example 1. Consider on $\mathbb{R}$ the differential equation:

$$
\begin{aligned}
& \left((\sin 8 t+2)\left(x^{\prime}(t)\right)^{\gamma}\right)^{\prime}+c_{0} t \sin ^{2} 8 t x^{\gamma}(t) \\
& \quad+4 c_{1} t^{2} \cos 2 t|x(t)|^{5 \gamma / 2} \operatorname{sgn} x(t) \\
& \quad+c_{2} t \sin 2 t \operatorname{sgn}|x(t)|^{\gamma / 2}=-\cos 4 t, \quad t \geq 1 .
\end{aligned}
$$

We set

$$
\begin{gathered}
r(t)=\sin 8 t+2, \quad q_{0}(t)=c_{0} t \sin ^{2} 8 t, \quad q_{1}(t)=4 c_{1} t^{2} \cos 2 t, \\
q_{2}(t)=c_{2} t \sin 2 t, \quad e(t)=\cos 4 t, \quad \delta_{i}(t)=t(i=0,1,2), \\
\alpha_{0}=\gamma, \quad \alpha_{1}=\frac{5 \gamma}{2}, \quad \alpha_{2}=\frac{\gamma}{2}, \\
g_{0,1}^{\gamma}(t)=g_{1,1}^{\gamma}(t)=g_{2,1}^{\gamma}(t)=1 .
\end{gathered}
$$

Also, let $\eta_{i}=1 / 3$, and

$$
\begin{gathered}
a_{1}=2 h \pi, \quad b i=a_{2}=2 h \pi+\frac{\pi}{8}, \\
b_{2}=2 h \pi+\frac{\pi}{4}(h=1,2,3 \ldots),
\end{gathered}
$$

such that (32) and (33) hold

$$
\begin{array}{r}
q_{i}(t) \geq 0 \text { on }\left[2 h \pi, 2 h \pi+\frac{\pi}{8}\right) \cup\left[2 h \pi+\frac{\pi}{8}, 2 h \pi+\frac{\pi}{4}\right), \\
i=0,1,2, \\
(-1)^{k} e(t) \geq 0, \quad t \in\left[2 h \pi, 2 h \pi+\frac{\pi}{8}\right) \cup\left[2 h \pi+\frac{\pi}{8}, 2 h \pi+\frac{\pi}{4}\right), \\
k=1,2 .
\end{array}
$$

Setting $u(t)=\sin 8 t$, we get $Q_{1,1}(t)=t\left(c_{0} \sin ^{8} t+\right.$ $\left.3\left(2 c_{1} c_{2} \sin 4 t|\cos 4 t|\right)^{1 / 3}\right)$. By $(42)$, we have

$$
\begin{gathered}
\int_{a_{k}}^{b_{k}}\left[\left(u^{\sigma}(s)\right)^{\gamma+1} Q_{1, k}(s)-\left(u^{\Delta}(s)\right)^{\gamma+1} r(s)\right] d s \\
=\int_{2 \pi}^{2 \pi+\pi / 8}\left[\sin ^{\gamma+1} 8 t \cdot t\left(c_{0} \sin ^{2} 8 t+3\left(2 c_{1} c_{2} \sin 4 t|\cos 4 t|\right)^{1 / 3}\right)\right. \\
\left.-(8 \cos 8 t)^{\gamma+1}(\sin 8 t+2)\right] d t \\
\geq \int_{0}^{\pi / 8}\left[2 \pi \sin ^{\gamma+1} 8 t\left(c_{0} \sin ^{2} 8 t+3\left(c_{1} c_{2} \sin 8 t\right)^{1 / 3}\right)\right. \\
\left.-8^{\gamma}\left(2 \cos ^{\gamma+1} 8 t+\sin 8 t \cos ^{\gamma+1} 8 t\right)\right] d t
\end{gathered}
$$

$$
\begin{aligned}
& =\frac{\pi \sqrt{\pi}}{4}\left[\frac{c_{0} \gamma(\gamma+2) \Gamma(\gamma / 2)}{(\gamma+3)(\gamma+1) \Gamma((\gamma+1) / 2)}\right. \\
& \left.\quad+\frac{3 \sqrt[3]{c_{1} c_{2}}(3 \gamma+1) \Gamma((3 \gamma+1) / 6)}{3 \gamma+4 \Gamma((3 \gamma+4) / 6)}\right] \\
& \geq 8^{\gamma}\left[\frac{\gamma \sqrt{\pi} \Gamma(\gamma / 2)}{(\gamma+1) \Gamma((\gamma+1) / 2)}+\frac{1}{\gamma+2}\right]>0 .
\end{aligned}
$$

Hence, by Theorems $8,(83)$ is oscillatory.

Example 2. Consider on $\mathbb{Z}$ the equation:

$$
\begin{aligned}
& \Delta\left((\Delta x(t))^{3}\right)+A|x(t)|^{3} \operatorname{sgn}(x(t)) \\
& +B|x(t+2)|^{4} \operatorname{sgn}(x(t+2)) \\
& \quad+C|x(t+1)|^{2} \operatorname{sgn}(t+1)=e(t),
\end{aligned}
$$

where $t \in 0,1,2, \ldots, A, B, C>0$,

$$
e(t)=f(n)= \begin{cases}0, & t=8 j, 8 j+4, \\ -1, & t=8 j+1,8 j+2,8 j+3 \\ 1 & t=8 j+5,8 j+6,8 j+7 .\end{cases}
$$

We set $q_{0}(t)=A, q_{1}(t)=B, q_{2}(t)=C, \alpha_{0}=\gamma=3, \alpha_{1}=4$, $\alpha_{2}=2, \delta_{0}(t)=t, \delta_{1}(t)=t+2, \delta_{2}(t)=t+1$.

Setting $a_{1}=8 j, b_{1}=8 j+2$, we can get $G_{1}\left(a_{1}\right)=8 j$, $G_{2}\left(b_{1}\right)=8 j+4$.

So, $e(t)$ satisfies the assumption in Theorem 9. Let $\eta_{1}=$ $\eta_{2}=1 / 2$ such that (32) and (34) hold. Setting $u(t)=t \bmod 2$, we get $Q_{2,1}(8 j)=(8 / 9) \sqrt{B C}$. By $(58)$, we get

$$
\begin{aligned}
\int_{a_{1}}^{b_{1}} & {\left[\left(u^{\sigma}(s)\right)^{\gamma+1} Q_{2, k}(s)-\left(u^{\Delta}(s)\right)^{\gamma+1} r(s)\right] \Delta s } \\
& =\sum_{t=8 j}^{8 j+1}\left(u^{4}(t+1) Q_{2,1}(t)-(u(t+1)-u(t))^{4}\right) \\
& =-2+\frac{8}{9} \sqrt{B C} .
\end{aligned}
$$

Consequently, if the constant coefficients $A, B, C>0$ satisfy the relation

$$
\frac{8}{9} \sqrt{B C} \geq 2
$$

then the above sum is nonnegative and hence (88) is oscillatory by Theorem 9 .

\section{Conflict of Interests}

The authors declare that there is no conflict of interests regarding the publication of this paper.

\section{Acknowledgments}

The authors sincerely thank the referees for their valuable comments and suggestions. This project is supported by 
the NNSF of China (no. 11271379), the Science Foundation of Guangdong University of Finance (no. 13XJ03-05), and the Foundation for Technology Innovation in Higher Education of Guangdong, China (no. 2013KJCX0136).

\section{References}

[1] S. H. Saker, "Boundedness of solutions of second-order forced nonlinear dynamic equations," The Rocky Mountain Journal of Mathematics, vol. 36, no. 6, pp. 2027-2039, 2006.

[2] Y. G. Sun and J. S. W. Wong, "Oscillation criteria for second order forced ordinary differential equations with mixed nonlinearities," Journal of Mathematical Analysis and Applications, vol. 334, no. 1, pp. 549-560, 2007.

[3] L. Erbe, A. Peterson, and S. H. Saker, "Oscillation criteria for a forced second-order nonlinear dynamic equation," Journal of Difference Equations and Applications, vol. 14, no. 10-11, pp. 9971009, 2008.

[4] C. Li and S. Chen, "Oscillation of second-order functional differential equations with mixed nonlinearities and oscillatory potentials," Applied Mathematics and Computation, vol. 210, no. 2, pp. 504-507, 2009.

[5] Q. Lin, B. Jia, and Q. Wang, "Forced oscillation of secondorder half-linear dynamic equations on time scales," Abstract and Applied Analysis, vol. 2010, Article ID 294194, 10 pages, 2010.

[6] L. Erbe, T. S. Hassan, A. Peterson, and S. H. Saker, "Interval oscillation criteria for forced second-order nonlinear delay dynamic equations with oscillatory potential," Dynamics of Continuous, Discrete \& Impulsive Systems A, vol. 17, no. 4, pp. 533-542, 2010.

[7] T. S. Hassan, L. Erbe, and A. Peterson, "Forced oscillation of second order differential equations with mixed nonlinearities," Acta Mathematica Scientia B, vol. 31, no. 2, pp. 613-626, 2011.

[8] D. R. Anderson and S. H. Saker, "Interval oscillation criteria for forced Emden-Fowler functional dynamic equations with oscillatory potential," Science China. Mathematics, vol. 56, no. 3, pp. 561-576, 2013.

[9] M. Bohner and A. Peterson, Dynamic Equations on Time Scales: An Introduction with Applications, Birkhäuser, Boston, Mass, USA, 2001.

[10] R. P. Agarwal and A. Zafer, "Oscillation criteria for secondorder forced dynamic equations with mixed nonlinearities," Advances in Difference Equations, vol. 2009, Article ID 938706, 20 pages, 2009.

[11] S. H. Saker, R. P. Agarwal, and D. O’Regan, “Oscillation results for second-order nonlinear neutral delay dynamic equations on time scales," Applicable Analysis, vol. 86, no. 1, pp. 1-17, 2007.

[12] S.-Y. Zhang and Q.-R. Wang, "Oscillation of second-order nonlinear neutral dynamic equations on time scales," Applied Mathematics and Computation, vol. 216, no. 10, pp. 2837-2848, 2010.

[13] S. H. Saker, "Oscillation criteria for second-order quasilinear neutral functional dynamic equation on time scales," Nonlinear Oscillations, vol. 13, no. 3, pp. 407-428, 2010.

[14] Y. B. Sun, Z. L. Han, T. X. Li, and G. R. Zhang, "Oscillation criteria for second-order quasilinear neutral delay dynamic equations on time scales," Advances in Difference Equations, vol. 2010, Article ID 512437, 14 pages, 2010.

[15] S.-Y. Zhang and Q.-R. Wang, "Oscillation criteria for secondorder nonlinear dynamic equations on time scales," Abstract and Applied Analysis, vol. 2012, Article ID 743469, 20 pages, 2012.

[16] R. M. Mathsen, Q.-R. Wang, and H.-W. Wu, "Oscillation for neutral dynamic functional equations on time scales," Journal of Difference Equations and Applications, vol. 10, no. 7, pp. 651659, 2004.

[17] Z.-Q. Zhu and Q.-R. Wang, "Frequency measures on time scales with applications," Journal of Mathematical Analysis and Applications, vol. 319, no. 2, pp. 398-409, 2006.

[18] Z.-Q. Zhu and Q.-R. Wang, "Existence of nonoscillatory solutions to neutral dynamic equations on time scales," Journal of Mathematical Analysis and Applications, vol. 335, no. 2, pp. 751762, 2007.

[19] H. Huang and Q.-R. Wang, "Oscillation of second-order nonlinear dynamic equations on time scales," Dynamic Systems and Applications, vol. 17, no. 3-4, pp. 551-570, 2008.

[20] Z.-H. Yu and Q.-R. Wang, "Asymptotic behavior of solutions of third-order nonlinear dynamic equations on time scales," Journal of Computational and Applied Mathematics, vol. 225, no. 2, pp. 531-540, 2009.

[21] E. F. Beckenbach and R. Bellman, Inequalities, Ergebnisse der Mathematik und ihrer Grenzgebiete, Springer, Berlin, Germany, 1961. 


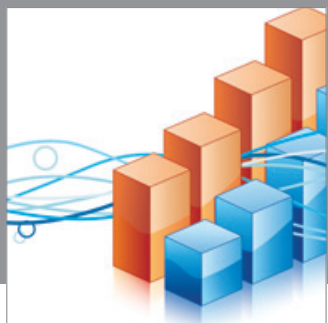

Advances in

Operations Research

mansans

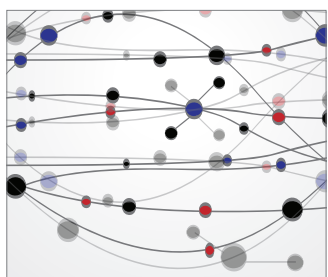

The Scientific World Journal
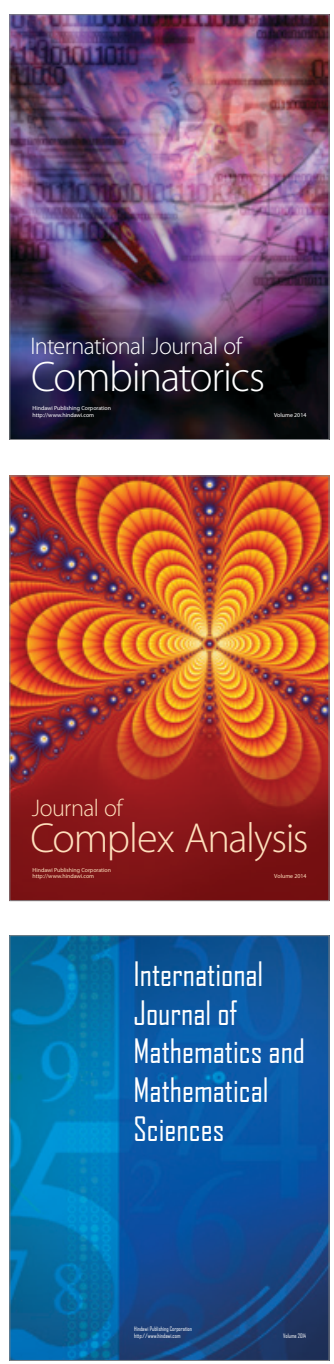
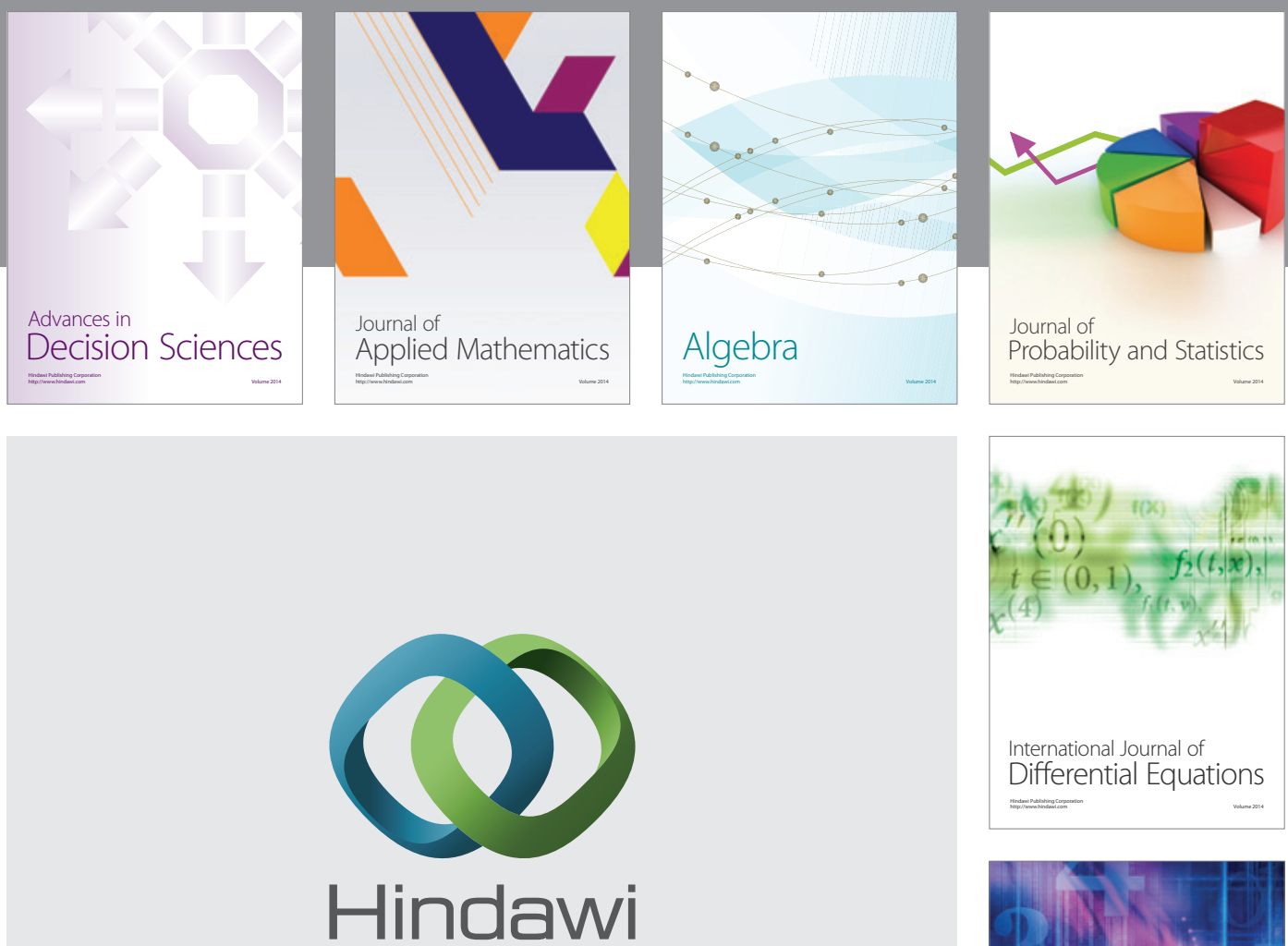

Submit your manuscripts at http://www.hindawi.com
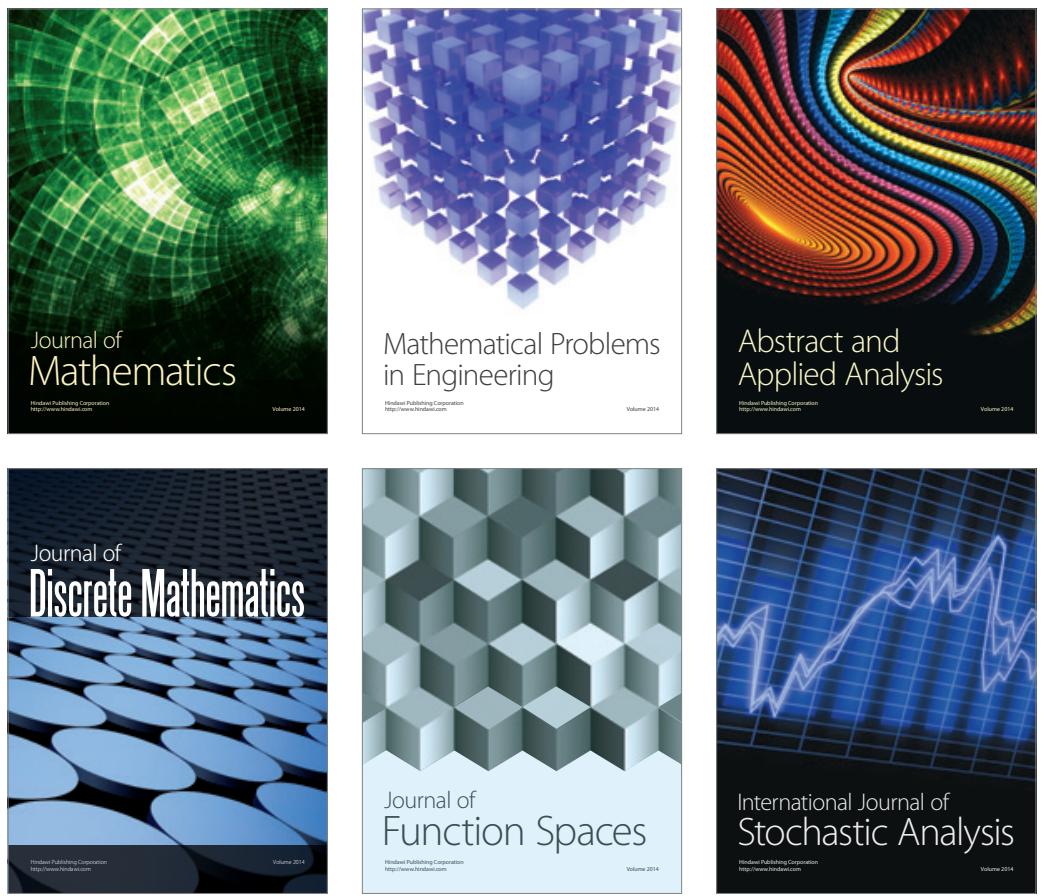

Journal of

Function Spaces

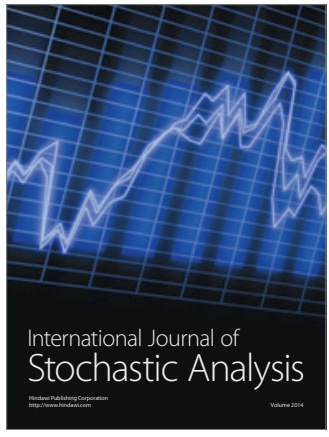

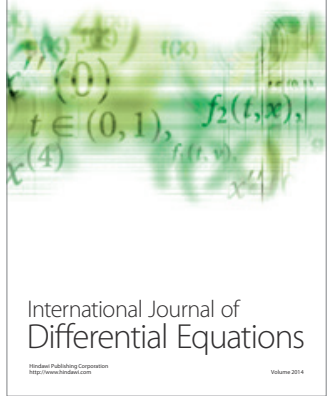
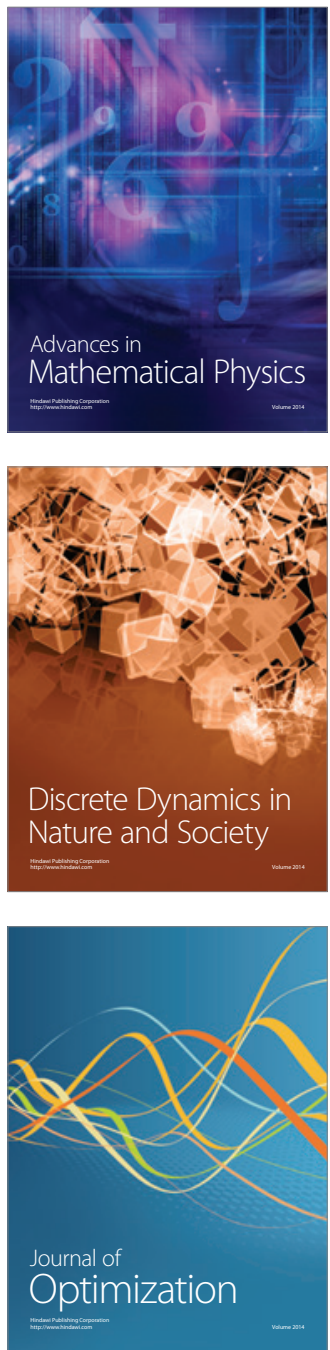